Article

\title{
Which Cryptocurrencies Are Mostly Traded in Distressed Times?
}

\author{
Nikolaos A. Kyriazis * and Paraskevi Prassa \\ Department of Economics, University of Thessaly, 28th October 78 Street, 38333 Volos, Greece \\ * Correspondence: knikolaos@uth.gr
}

Received: 10 July 2019; Accepted: 14 August 2019; Published: 20 August 2019

check for updates

\begin{abstract}
This paper investigates the level of liquidity of digital currencies during the very intense bearish phase in their markets. The data employed span the period from April 2018 until January 2019, which is the second phase of bearish times with almost constant decreases. The Amihud's illiquidity ratio is employed in order to measure the liquidity of these digital assets. Findings indicate that the most popular cryptocurrencies exhibit higher levels of liquidity during stressed periods. Thereby, it is revealed that investors' preferences for trading during highly risky times are favorable for well-known virtual currencies in the detriment of less-known ones. This enhances findings of relevant literature about strong and persistent positive or negative herding behavior of investors based on Bitcoin, Ethereum and highly-capitalized cryptocurrencies in general. Notably though, a tendency towards investing in the TrueUSD stablecoin has also emerged.
\end{abstract}

Keywords: Bitcoin; cryptocurrency; liquidity

JEL Classification: E44; G11; G12; G15

\section{Introduction}

Bitcoin and other digital currencies have spurred interest for research since late 2016 due to their increasing attractiveness as an investment tool. This has brought about a proliferating bulk of high-quality relevant academic papers (inter alia: Dyhrberg 2016a, 2016b; Katsiampa 2017; Corbet et al. 2018, 2019; Beneki et al. 2019; Kyriazis 2019). Cryptocurrencies are highly innovative means of transactions that have gained increasing popularity among investors and especially speculators seeking high profits, despite risk. Nevertheless, the functions of digital currencies as means for store of value and a unit of account are far from being established in the perceptions of economic agents. Nevertheless, the innovative character, the non-complexity and the lack of opacity in such currencies has led to the flourishing of issuance of new cryptocurrencies.

Decentralized transaction systems with blockchain technology have proven very promising for economic units of various risk appetites. Investments in digital currencies are considered to be extremely volatile although amazingly profitable in bullish periods. Investment and profit-making opportunities have also been apparent during bearish markets due to the high volatility of digital currencies. Emphasis should be paid to the fact that a small number of virtual coins that are most popular for investors make up a very large portion of market capitalization in the cryptocurrency markets.

This study builds on Wei (2018) which was the first academic paper investigating the liquidity levels of a very wide range of digital currencies. There are two main research hypotheses tested in this paper. Firstly, it is examined whether popular cryptocurrencies such as Bitcoin, Ethereum, Monero, Dash, Cardano and Steem are preferable by investors in bearish conditions in the cryptocurrency markets. Thereby, we investigate how the popularity and the incumbent character of specific coins in the virtual currency markets affect decision-making about active trading. 
The remainder of this study is organized as follows: Section 2 provides the main literature concerning digital currencies up to the present. Section 3 presents the data and methodology employed in order to conduct estimations about liquidity levels. Section 4 lays out and discusses the results as well as analyzes the economic implications. Finally, Section 5 concludes.

\section{Literature Review}

There has been a proliferating bulk of high-quality academic studies that investigate the factors determining performance of digital currencies. Ammous (2018) looks into whether Bitcoin, Ethereum, Litecoin, Ripple or Steem can fulfil the functions of money. He argues that only Bitcoin can be used as a store of value because of its commitment to increase its supply only by predetermined amounts. All cryptocurrencies are found to be inappropriate as units of account due to their large fluctuations. Moreover, theoretically, they can serve as a medium of exchange, but it is unlikely that they will become popular for this function. Bitcoin is found to be the most promising to function as money.

Brandvold et al. (2015) investigate how Bitcoin exchanges contribute to price discovery from April 2013 to February 2014. The unobserved components discovery model they employ leads to results supporting that MtGox was the main determinant of the price discovery process until its end. Furthermore, the Bitcoin's information share was found to be high, reflecting the increasing Chinese interest in Bitcoin. Overall, the information share is found to have been dynamic and evolve over time. Baur et al. (2018) investigate whether Bitcoin should be best considered as a medium of exchange or an asset for speculation by adopting data from July 2010 to June 2015. They argue that it is not a safe haven due to its weak correlation with conventional assets, such as stocks, bonds and commodities in normal times but also during crises. Findings indicate that Bitcoin is mostly used for speculative trading rather than as a medium of exchange and a new form of currency. By another perspective, Hayes (2017) investigates the determinants for value formation of cryptocurrencies by employing cross-sectional analysis for sixty-six of the most used digital currencies. He reveals that the level of competition in the network of producers, the rate of unit production as well as how difficult is the algorithm for mining are determinants of cryptocurrency value.

Bouri et al. (2017) examine how Bitcoin has affected global uncertainty during the period from March 2011 to October 2016, by employing the World VIX as a measure of uncertainty. The wavelet-based quantile-in-quantile methodology employed provides evidence that Bitcoin acts as a hedger against global uncertainty. This is valid especially in short investment horizons for both lower and upper quantiles of Bitcoin returns and uncertainty. Balcilar et al. (2017) utilize a Generalized Autoregressive Conditional Heteroskedasticity $(\mathrm{GARCH})$ methodology and a non-parametric causality-in-quantiles test for examining the causal nexus between trading volume and Bitcoin returns and volatility. Volume is found to be able to predict returns but not volatility at any point of the conditional distribution. However, the former is not true in Bitcoin bear and bull markets. It is also found that non-linearities are important for explaining behavior in tails regarding the causal nexus of Bitcoin returns with trading volume. Bitcoin trading based on the volume-return linkage can prove profitable. Furthermore, Pieters and Vivanco (2017) investigate whether Bitcoin prices follow the Rule of One Price across markets by evaluating eleven Bitcoin exchanges from June 2014 to July 2015. Findings indicate that failure of this rule is connected with markets where no ID is needed to fund an account, as they exhibit larger price deviations. Thereby, know-your-customer regulations can lead to a sizable effect on Bitcoin market. Further exploration of determinants of digital currency prices includes Gandal et al. (2018). They investigate the effect of suspicious trading activity on the Mt. Bitcoin currency exchange, in which almost 600,000 BTC were fraudulently acquired. They argue that this activity led to the spike of BTC price in late 2013, as trading volume increased significantly on those trading days. They support that a lack of regulations in markets lead to vulnerability regarding manipulation by speculators today.

Feng et al. (2018) propose a novel indicator for estimating the informed trades ahead of events related to cryptocurrencies and provide evidence that informed trading takes place in the Bitcoin 
market before such events. The preference of such traders is revealed for taking their positions two days before large positive events and one day before large negative events. This triggers large profits from trading. Corbet et al. (2018) employ a generalized variance decomposition methodology to measure the direction and intensity of spillovers across Bitcoin, Ripple, Litecoin and other selected markets. There is evidence that cryptocurrency markets are interconnected with each other and present similar patterns of connectedness with other important asset categories. Furthermore, Panagiotidis et al. (2018) examine the determinants of Bitcoin returns from June 2010 to June 2017 by adopting a Least Absolute Shrinkage and Selection Operator (LASSO) approach. Evidence uncovers that uncertainty negatively influences returns whereas currency exchange rates as well as interest rates, gold and oil have positive effects. The predicted signs are found for information demand and mixed impacts are traced from stock markets. Empirical outcomes reveal that search intensity and gold returns are the most influential. Amid literature on price determinants is Kim (2017) who examines the transaction cost of Bitcoin from April 2014 to April 2015. He argues that the bid-ask spreads of Bitcoin exchanges are lower than those of the retail foreign exchange market. This cost advantage equals $5 \%$ and is due to the simpler infrastructure and the efficiency that characterizes the Bitcoin market.

In another vein, Adhami et al. (2018) analyze 253 Initial Coin Offerings (ICOs) of cryptocurrencies from August 2014 to August 2017 in order to reveal the determinants of their success. They document that when the code source is available, a token presale is organized and tokens allow contributors to access a specific service or to share profits, then success is higher. Bouri et al. (2018) employ the Generalized Supremum Augmented Dickey Fuller (GSADF) test in order to study co-explosivity in cryptocurrency markets. They detect multiple periods of explosivity, especially during the 2017 bullish market and this is more intense concerning Bitcoin. Explosivity in a digital currency market is found to affect explosivity in markets of other virtual coins. Moreover, Bouri et al. (2019b) reveal that the market of cryptocurrencies presents time-varying herding behavior. High comovement is detected in the cross-sectional returns' dispersion across digital currency markets. This implies that mimicking of others' decisions takes place by investors in digital currency markets.

Schilling and Uhlig (2019) present a model of an endowment economy with the US dollar and Bitcoin constituting two competing but intrinsically worthless currencies. They support that fluctuations in prices are not harmful for the medium-of-exchange function of Bitcoin. Moreover, they support that if all economic units were impatient, no speculation opportunities would occur in the Bitcoin market. Giudici and Abu-Hashish (2019) adopt a correlation network Vector Autoregressive (VAR) process and provide evidence that Bitcoin prices from different exchanges are highly interrelated and that prices from larger exchanges drive prices in smaller ones. Furthermore, they support that the inclusion of Bitcoin in portfolios results in diversification benefits. Ji et al. (2019) investigate the spillovers among six major virtual currencies by building positive and negative returns-connectedness and volatility-connectedness networks. They argue that leading digital currencies are interconnected and that Litecoin exerts a significant impact on Bitcoin and other digital coins of primary importance. Asymmetries in spillovers are also detected and are larger in negative-return spillovers than in positive-returns impacts. Kyriazis et al. (2019) examine whether high-capitalization cryptocurrencies are affected by Bitcoin, Ethereum and Ripple during bearish times by employing a number of Generalized Autoregressive Conditional Heteroskedasticty (GARCH) specifications. Evidence reveals that the majority of digital coins investigated are complementary with the three drivers of the market and that no hedging abilities exist.

Bouri et al. (2019a) examine the persistence in the level and volatility of Bitcoin prices by taking into consideration the impact of structural breaks. They argue for the existence of long-memory in volatility of Bitcoin and identify structural changes in the dynamics of this leading digital currency. Moreover, Bouri et al. (2019c) investigate the ability of trading volume of seven major digital currencies on predicting their returns and volatility. They provide evidence that trading volume triggers extreme negative and positive effects of all currencies under scrutiny whereas only influences the volatility of Litecoin, Nem and Dash. Ferreira and Pereira (2019) examine the contagion effects in markets 
of virtual currencies by employing detrended cross-correlation analysis coefficients. They provide evidence that the contagion effect between Bitcoin and other digital coins has been more intense and that the digital currency market has been more integrated during bearish times. Hyun et al. (2019) examine dependency among Bitcoin, Ethereum, Litecoin, Ripple and Stellar using a copula directional dependence (CDD) approach. They provide evidence that dependency from Bitcoin to Litecoin is the highest one and that dependency from Ethereum to the other four currencies is higher than the other way around. By their own perspective, Dastgir et al. (2019) investigate the causality between the Google Trends search queries and returns of Bitcoin from January 2013 to December 2017, by applying a Copula-based Granger causality in Distribution (GCCD) test. They document that a bi-directional causal nexus between Bitcoin attention and Bitcoin returns exists mainly in the left tail (bad performance) and the right tail (superior performance) of the distribution, but not in the central distributions. In a somewhat similar vein, Shen et al. (2019) use the number of tweets from Twitter as a measure of attention and examine their influence on Bitcoin. They argue that tweets constitute a significant determinant of the next day's trading volume and realized volatility of Bitcoin.

\section{Data and Methodology}

This study has undertaken the strenuous task of investigating the level of liquidity of cryptocurrencies during the second phase of bearish behavior in the digital coins market. In order to provide the most representative view of liquidity in virtual currencies, we have downloaded, from the reliable source of coinmarketcap.com, the full spectrum of such currencies that existed on 12 February 2019. This has led to a total number of over 1900 coins from which we have short-listed only those that did not have gaps in the time series of their quotes. Thereby, the final sample consists of 846 digital currencies that cover the extra-bearish period of 1 April 2018 to 31 January 2019. All quotes about prices and trading volume are in daily frequencies.

The methodology employed for our estimations is based on the well-known Amihud's illiquidity ratio based on Amihud (2002), which takes the following form:

$$
\text { Illiquidity }_{T}^{i}=\frac{1}{D_{T}} \sum_{t=1}^{D_{T}} \frac{\left|R_{t}^{i}\right|}{P_{t}^{i} V_{t}^{i}}
$$

where $D_{T}$ represents the number of traded days during the period examined, $R_{t, T}^{i}$ stands for the daily return of digital currency $i$ on day $t, V_{t}^{i}$ is the volume traded of asset $i$ in day $t$ and $P_{t}^{i}$ represents the daily price of cryptocurrency $i$ on day $t$. All market prices of digital coins are expressed in relation to US dollars. It should be noted that the currencies that exhibit low values of the Amihud's illiquidity ratio are considered to the most liquid.

\section{Empirical Results}

In Tables 1 and 2, we categorize cryptocurrencies into eight groups based on the Amihud's illiquidity ratio in order to detect investors' trading preferences during the distressed bearish period investigated. The first group represents the most liquid digital currencies whereas the eighth group is comprised by the least liquid ones. 
Table 1. Groups of most liquid cryptocurrencies.

\begin{tabular}{|c|c|c|c|c|c|c|c|}
\hline \multicolumn{2}{|c|}{ Group 1 (Most Liquid) } & \multicolumn{2}{|c|}{ Group 2} & \multicolumn{2}{|c|}{ Group 3} & \multicolumn{2}{|c|}{ Group 4} \\
\hline Names & $\begin{array}{l}\text { Amihud } \\
\text { Illiquidity } \\
\text { Ratio }\end{array}$ & Names & $\begin{array}{c}\text { Amihud } \\
\text { Illiquidity } \\
\text { Ratio }\end{array}$ & Names & $\begin{array}{l}\text { Amihud } \\
\text { Illiquidity } \\
\text { Ratio }\end{array}$ & Names & $\begin{array}{c}\text { Amihud } \\
\text { Illiquidity } \\
\text { Ratio }\end{array}$ \\
\hline Tether & $9.09 \times 10^{-28}$ & Storj & $5.72 \times 10^{-12}$ & Ignis & $6.62 \times 10^{-11}$ & SIBCoin & $3.39 \times 10^{-10}$ \\
\hline Bitcoin & $2.3 \times 10^{-19}$ & Tezos & $5.73 \times 10^{-12}$ & FirstBlood & $6.74 \times 10^{-11}$ & Syndicate & $3.41 \times 10^{-10}$ \\
\hline Ethereum & $2 \times 10^{-17}$ & United Bitcoin & $5.76 \times 10^{-12}$ & ZrCoin & $7 \times 10^{-11}$ & Bezop & $3.43 \times 10^{-10}$ \\
\hline Stellar & $3.47 \times 10^{-17}$ & Loom Network & $5.78 \times 10^{-12}$ & TaaS & $7.05 \times 10^{-11}$ & IXT & $3.45 \times 10^{-10}$ \\
\hline Bitcoin Cash & $4.73 \times 10^{-17}$ & Loopring & $5.84 \times 10^{-12}$ & MinexCoin & $7.11 \times 10^{-11}$ & PayPie & $3.46 \times 10^{-10}$ \\
\hline IOTA & $5.14 \times 10^{-16}$ & Power Ledger & $5.88 \times 10^{-12}$ & GameCredits & $7.29 \times 10^{-11}$ & DecentBet & $3.48 \times 10^{-10}$ \\
\hline Tether & $5.14 \times 10^{-16}$ & BitShares & $6.1 \times 10^{-12}$ & $\begin{array}{l}\text { Molecular } \\
\text { Future }\end{array}$ & $7.47 \times 10^{-11}$ & BlackCoin & $3.49 \times 10^{-10}$ \\
\hline Zcash & $9.34 \times 10^{-16}$ & iExec RLC & $6.13 \times 10^{-12}$ & Siacoin & $7.48 \times 10^{-11}$ & Acute Angle C & $3.51 \times 10^{-10}$ \\
\hline EOS & $1.21 \times 10^{-15}$ & bitUSD & $6.27 \times 10^{-12}$ & Dynamic & $7.61 \times 10^{-11}$ & KickCoin & $3.55 \times 10^{-10}$ \\
\hline $\begin{array}{l}\text { Ethereum } \\
\text { Classic }\end{array}$ & $5.28 \times 10^{-15}$ & Ark & $6.34 \times 10^{-12}$ & Bottos & $7.86 \times 10^{-11}$ & BridgeCoin & $3.63 \times 10^{-10}$ \\
\hline NEO & $5.73 \times 10^{-15}$ & Emercoin & $6.54 \times 10^{-12}$ & $\begin{array}{l}\text { Genaro } \\
\text { Network }\end{array}$ & $7.91 \times 10^{-11}$ & Playkey & $3.8 \times 10^{-10}$ \\
\hline Dai & $6.28 \times 10^{-15}$ & IoT Chain & $6.77 \times 10^{-12}$ & NAGA & $8.19 \times 10^{-11}$ & ReddCoin & $3.84 \times 10^{-10}$ \\
\hline Maker & $6.58 \times 10^{-15}$ & Polymath & $6.96 \times 10^{-12}$ & Quantstamp & $8.31 \times 10^{-11}$ & ETHLend & $3.87 \times 10^{-10}$ \\
\hline Binance Coin & $1.04 \times 10^{-14}$ & Exchange Union & $7 \times 10^{-12}$ & SONM & $8.32 \times 10^{-11}$ & Aeon & $3.89 \times 10^{-10}$ \\
\hline Qtum & $1.76 \times 10^{-14}$ & Numeraire & $7.11 \times 10^{-12}$ & Stox & $8.43 \times 10^{-11}$ & RevolutionVR & $3.92 \times 10^{-10}$ \\
\hline Bitcoin Gold & $2.91 \times 10^{-14}$ & Chronobank & $7.19 \times 10^{-12}$ & Qbao & $8.43 \times 10^{-11}$ & $\begin{array}{l}\text { DeepBrain } \\
\text { Chain }\end{array}$ & $3.94 \times 10^{-10}$ \\
\hline Augur & $3.35 \times 10^{-14}$ & Viacoin & $7.57 \times 10^{-12}$ & DADI & $8.47 \times 10^{-11}$ & Paragon & $3.95 \times 10^{-10}$ \\
\hline Waves & $3.44 \times 10^{-14}$ & Blocknet & $8.66 \times 10^{-12}$ & Phore & $8.84 \times 10^{-11}$ & HollyWoodCoin & $4.03 \times 10^{-10}$ \\
\hline Decred & $3.67 \times 10^{-14}$ & Nexus & $8.68 \times 10^{-12}$ & Rcoin & $8.93 \times 10^{-11}$ & DeepOnion & $4.1 \times 10^{-10}$ \\
\hline Huobi Token & $4.74 \times 10^{-14}$ & $\begin{array}{c}\text { Raiden } \\
\text { Networ... }\end{array}$ & $8.76 \times 10^{-12}$ & SingularityNET & $9.07 \times 10^{-11}$ & LatiumX & $4.11 \times 10^{-10}$ \\
\hline WETH & $4.8 \times 10^{-14}$ & Vertcoin & $9.29 \times 10^{-12}$ & Ink & $9.66 \times 10^{-11}$ & DEW & $4.3 \times 10^{-10}$ \\
\hline 42-coin & $5.62 \times 10^{-14}$ & Santiment Net & $9.78 \times 10^{-12}$ & Bitcoin Green & $9.79 \times 10^{-11}$ & Crown & $4.42 \times 10^{-10}$ \\
\hline DigixDAO & $5.82 \times 10^{-14}$ & $\begin{array}{l}\text { SIRIN LABS } \\
\text { Token }\end{array}$ & $9.79 \times 10^{-12}$ & Expanse & $9.81 \times 10^{-11}$ & Profile Utili & $4.53 \times 10^{-10}$ \\
\hline OmiseGO & $6.33 \times 10^{-14}$ & Status & $1.01 \times 10^{-11}$ & Espers & $9.81 \times 10^{-11}$ & Blox & $4.59 \times 10^{-10}$ \\
\hline Moeda Loyalty & $6.93 \times 10^{-14}$ & Decentraland & $1.01 \times 10^{-11}$ & LEOcoin & $9.86 \times 10^{-11}$ & FLO & $4.6 \times 10^{-10}$ \\
\hline Project- $X$ & $9.21 \times 10^{-14}$ & Smartlands & $1.02 \times 10^{-11}$ & OST & $9.88 \times 10^{-11}$ & Gambit & $4.66 \times 10^{-10}$ \\
\hline TrueChain & $1.02 \times 10^{-13}$ & AdEx & $1.04 \times 10^{-11}$ & VIBE & $1.02 \times 10^{-10}$ & WhiteCoin & $4.87 \times 10^{-10}$ \\
\hline MobileGo & $1.48 \times 10^{-13}$ & Verge & $1.09 \times 10^{-11}$ & Republic Prot & $1.05 \times 10^{-10}$ & TopChain & $4.9 \times 10^{-10}$ \\
\hline TRON & $2.28 \times 10^{-13}$ & Blocktix & $1.2 \times 10^{-11}$ & CPChain & $1.06 \times 10^{-10}$ & DATA & $4.97 \times 10^{-10}$ \\
\hline Lisk & $2.69 \times 10^{-13}$ & Bodhi & $1.22 \times 10^{-11}$ & Pundi $X$ & $1.07 \times 10^{-10}$ & Anoncoin & $5.05 \times 10^{-10}$ \\
\hline Genesis Vision & $3.16 \times 10^{-13}$ & $\begin{array}{l}\text { Global Tour } \\
\text { Coin }\end{array}$ & $1.25 \times 10^{-11}$ & adToken & $1.08 \times 10^{-10}$ & REBL & $5.09 \times 10^{-10}$ \\
\hline bitCNY & $3.27 \times 10^{-13}$ & Everex & $1.28 \times 10^{-11}$ & Kcash & $1.09 \times 10^{-10}$ & SmartCash & $5.27 \times 10^{-10}$ \\
\hline Groestlcoin & $3.35 \times 10^{-13}$ & Delphy & $1.29 \times 10^{-11}$ & $\begin{array}{c}\text { Streamr } \\
\text { DATAcoin }\end{array}$ & $1.09 \times 10^{-10}$ & Swarm & $5.29 \times 10^{-10}$ \\
\hline Metaverse ETP & $3.38 \times 10^{-13}$ & KuCoin Shares & $1.31 \times 10^{-11}$ & RChain & $1.11 \times 10^{-10}$ & Patientory & $5.57 \times 10^{-10}$ \\
\hline Ontology & $3.43 \times 10^{-13}$ & Matrix AI Net & $1.34 \times 10^{-11}$ & $\begin{array}{l}\text { Request } \\
\text { Network }\end{array}$ & $1.12 \times 10^{-10}$ & BOScoin & $5.67 \times 10^{-10}$ \\
\hline Lunyr & $3.89 \times 10^{-13}$ & Civic & $1.34 \times 10^{-11}$ & DubaiCoin & $1.12 \times 10^{-10}$ & Bulwark & $5.75 \times 10^{-10}$ \\
\hline ICON & $4.15 \times 10^{-13}$ & Factom & $1.39 \times 10^{-11}$ & Quantum Resis & $1.17 \times 10^{-10}$ & GET Protocol & $5.79 \times 10^{-10}$ \\
\hline HorizenHorizen & $4.2 \times 10^{-13}$ & IOST & $1.47 \times 10^{-11}$ & SunContract & $1.18 \times 10^{-10}$ & YEE & $6.19 \times 10^{-10}$ \\
\hline Populous & $4.34 \times 10^{-13}$ & Bread & $1.49 \times 10^{-11}$ & TokenCard & $1.18 \times 10^{-10}$ & Hi Mutual Soc & $6.36 \times 10^{-10}$ \\
\hline $0 \mathrm{x}$ & $4.61 \times 10^{-13}$ & NavCoin & $1.58 \times 10^{-11}$ & Jibrel Network & $1.2 \times 10^{-10}$ & Everus & $6.41 \times 10^{-10}$ \\
\hline Nano & $4.71 \times 10^{-13}$ & Counterparty & $1.61 \times 10^{-11}$ & TransferCoin & $1.23 \times 10^{-10}$ & Upfiring & $6.47 \times 10^{-10}$ \\
\hline Gas & $4.85 \times 10^{-13}$ & ProChain & $1.62 \times 10^{-11}$ & district0x & $1.24 \times 10^{-10}$ & Elastic & $6.5 \times 10^{-10}$ \\
\hline Gnosis & $4.86 \times 10^{-13}$ & Monero & $1.68 \times 10^{-11}$ & NeosCoin & $1.28 \times 10^{-10}$ & LBRY Credits & $6.52 \times 10^{-10}$ \\
\hline Bibox Token & $4.96 \times 10^{-13}$ & Achain & $1.7 \times 10^{-11}$ & WePower & $1.28 \times 10^{-10}$ & Cofound.it & $6.55 \times 10^{-10}$ \\
\hline Aeternity & $5.2 \times 10^{-13}$ & Revain & $1.7 \times 10^{-11}$ & Scry.info & $1.3 \times 10^{-10}$ & ATN & $6.65 \times 10^{-10}$ \\
\hline SaluS & $5.22 \times 10^{-13}$ & Peercoin & $1.77 \times 10^{-11}$ & Suretly & $1.31 \times 10^{-10}$ & $\begin{array}{l}\text { Insights } \\
\text { Network }\end{array}$ & $7.6 \times 10^{-10}$ \\
\hline Stellar & $5.72 \times 10^{-13}$ & Substratum & $1.8 \times 10^{-11}$ & YOYOW & $1.31 \times 10^{-10}$ & Electroneum & $7.67 \times 10^{-10}$ \\
\hline Veritaseum & $5.76 \times 10^{-13}$ & Syscoin & $1.8 \times 10^{-11}$ & Global Curren & $1.33 \times 10^{-10}$ & Decision Token & $7.85 \times 10^{-10}$ \\
\hline $\mathrm{MCO}$ & $5.87 \times 10^{-13}$ & IPChain & $1.89 \times 10^{-11}$ & Polis & $1.35 \times 10^{-10}$ & HalalChain & $8.01 \times 10^{-10}$ \\
\hline
\end{tabular}


Table 1. Cont.

\begin{tabular}{|c|c|c|c|c|c|c|c|}
\hline \multicolumn{2}{|c|}{ Group 1 (Most Liquid) } & \multicolumn{2}{|c|}{ Group 2} & \multicolumn{2}{|c|}{ Group 3} & \multicolumn{2}{|c|}{ Group 4} \\
\hline Names & $\begin{array}{l}\text { Amihud } \\
\text { Illiquidity } \\
\text { Ratio }\end{array}$ & Names & $\begin{array}{l}\text { Amihud } \\
\text { Illiquidity } \\
\text { Ratio }\end{array}$ & Names & $\begin{array}{c}\text { Amihud } \\
\text { Illiquidity } \\
\text { Ratio }\end{array}$ & Names & $\begin{array}{l}\text { Amihud } \\
\text { Illiquidity } \\
\text { Ratio }\end{array}$ \\
\hline Waltonchain & $6.01 \times 10^{-13}$ & LockTrip & $1.93 \times 10^{-11}$ & Matryx & $1.36 \times 10^{-10}$ & Flixxo & $8.07 \times 10^{-10}$ \\
\hline ZClassic & $6.51 \times 10^{-13}$ & CRYPTO20 & $1.93 \times 10^{-11}$ & UpToken & $1.38 \times 10^{-10}$ & TerraNova & $8.08 \times 10^{-10}$ \\
\hline ZCoin & $6.85 \times 10^{-13}$ & MaidSafeCoin & $1.98 \times 10^{-11}$ & Bytecoin & $1.43 \times 10^{-10}$ & $\begin{array}{c}\text { ACE } \\
\text { (TokenStars) }\end{array}$ & $8.09 \times 10^{-10}$ \\
\hline Neblio & $7.22 \times 10^{-13}$ & Aragon & $1.98 \times 10^{-11}$ & CPChain & $1.46 \times 10^{-10}$ & RealChain & $8.18 \times 10^{-10}$ \\
\hline $\begin{array}{l}\text { Bitcoin } \\
\text { Diamond }\end{array}$ & $7.3 \times 10^{-13}$ & B2BX & $2.06 \times 10^{-11}$ & Bankex & $1.47 \times 10^{-10}$ & Zeepin & $8.22 \times 10^{-10}$ \\
\hline $\begin{array}{c}\text { IDEX } \\
\text { Membership }\end{array}$ & $7.42 \times 10^{-13}$ & POA Network & $2.15 \times 10^{-11}$ & Hacken & $1.51 \times 10^{-10}$ & CryptoPing & $8.29 \times 10^{-10}$ \\
\hline GXChain & $7.68 \times 10^{-13}$ & All Sports & $2.24 \times 10^{-11}$ & Solaris & $1.51 \times 10^{-10}$ & QunQun & $8.32 \times 10^{-10}$ \\
\hline Stratis & $7.94 \times 10^{-13}$ & Fargocoin & $2.35 \times 10^{-11}$ & Unikoin Gold & $1.58 \times 10^{-10}$ & Fortuna & $8.33 \times 10^{-10}$ \\
\hline Mithril & $7.95 \times 10^{-13}$ & Bitcoin God & $2.42 \times 10^{-11}$ & Tierion & $1.62 \times 10^{-10}$ & Memetic / Pep & $8.34 \times 10^{-10}$ \\
\hline aelf & $7.96 \times 10^{-13}$ & Ardor & $2.55 \times 10^{-11}$ & SmartMesh & $1.66 \times 10^{-10}$ & EZToken & $8.69 \times 10^{-10}$ \\
\hline SPINDLE & $8.04 \times 10^{-13}$ & Ormeus Coin & $2.84 \times 10^{-11}$ & Agrello & $1.73 \times 10^{-10}$ & ClubCoin & $8.73 \times 10^{-10}$ \\
\hline Wanchain & $8.23 \times 10^{-13}$ & Crypterium & $2.96 \times 10^{-11}$ & Red Pulse Pho... & $1.73 \times 10^{-10}$ & SportyCo & $8.86 \times 10^{-10}$ \\
\hline Bitmark & $8.26 \times 10^{-13}$ & TransferCoin & $2.97 \times 10^{-11}$ & Time New Bank & $1.76 \times 10^{-10}$ & $\begin{array}{c}\text { Banyan } \\
\text { Network }\end{array}$ & $9.14 \times 10^{-10}$ \\
\hline Skycoin & $8.57 \times 10^{-13}$ & Wings & $3.03 \times 10^{-11}$ & Nucleus Vision & $1.79 \times 10^{-10}$ & Mobius & $9.16 \times 10^{-10}$ \\
\hline Dash & $9.13 \times 10^{-13}$ & Dragonchain & $3.06 \times 10^{-11}$ & GoByte & $1.81 \times 10^{-10}$ & BitBay & $9.35 \times 10^{-10}$ \\
\hline Cardano & $9.13 \times 10^{-13}$ & iCoin & $3.15 \times 10^{-11}$ & Swarm City & $1.82 \times 10^{-10}$ & United Trader... & $9.58 \times 10^{-10}$ \\
\hline Bancor & $9.62 \times 10^{-13}$ & Iconomi & $3.15 \times 10^{-11}$ & Bloom & $1.82 \times 10^{-10}$ & Lamden & $9.62 \times 10^{-10}$ \\
\hline Steem & $9.91 \times 10^{-13}$ & Einsteinium & $3.16 \times 10^{-11}$ & Etheroll & $1.84 \times 10^{-10}$ & I/O Coin & $9.91 \times 10^{-10}$ \\
\hline CloakCoin & $1.29 \times 10^{-12}$ & Uquid Coin & $3.21 \times 10^{-11}$ & TrueFlip & $1.86 \times 10^{-10}$ & Radium & $9.91 \times 10^{-10}$ \\
\hline LiteBitcoin & $1.36 \times 10^{-12}$ & Internet Node & $3.26 \times 10^{-11}$ & MediShares & $1.87 \times 10^{-10}$ & Change & $1.01 \times 10^{-9}$ \\
\hline Nuls & $1.39 \times 10^{-12}$ & BnkToTheFuture & $3.27 \times 10^{-11}$ & Polybius & $1.89 \times 10^{-10}$ & EchoLink & $1.03 \times 10^{-9}$ \\
\hline Super Bitcoin & $1.55 \times 10^{-12}$ & QASH & $3.5 \times 10^{-11}$ & Po.et & $1.98 \times 10^{-10}$ & Stipend & $1.04 \times 10^{-9}$ \\
\hline AurumCoin & $1.6 \times 10^{-12}$ & Storm & $3.5 \times 10^{-11}$ & Pascal Coin & $2 \times 10^{-10}$ & VeriumReserve & $1.04 \times 10^{-9}$ \\
\hline Fusion & $1.64 \times 10^{-12}$ & WAX & $3.59 \times 10^{-11}$ & Privatix & $2.01 \times 10^{-10}$ & Open Trading & $1.04 \times 10^{-9}$ \\
\hline WaykiChain & $1.71 \times 10^{-12}$ & OAX & $3.71 \times 10^{-11}$ & Neumark & $2.06 \times 10^{-10}$ & Sumokoin & $1.13 \times 10^{-9}$ \\
\hline KingN Coin & $1.84 \times 10^{-12}$ & Ambrosus & $3.79 \times 10^{-11}$ & COss & $2.11 \times 10^{-10}$ & MyBit & $1.16 \times 10^{-9}$ \\
\hline Cryptonex & $1.87 \times 10^{-12}$ & Enjin Coin & $3.81 \times 10^{-11}$ & SingularDTV & $2.12 \times 10^{-10}$ & Hydro Protocol & $1.16 \times 10^{-9}$ \\
\hline Golem & $1.9 \times 10^{-12}$ & Credits & $3.94 \times 10^{-11}$ & SureRemit & $2.15 \times 10^{-10}$ & Humaniq & $1.18 \times 10^{-9}$ \\
\hline Particl & $1.9 \times 10^{-12}$ & Ubiq & $4.06 \times 10^{-11}$ & NetKoin & $2.17 \times 10^{-10}$ & Measurable Da & $1.19 \times 10^{-9}$ \\
\hline Metal & $1.97 \times 10^{-12}$ & Tokenomy & $4.12 \times 10^{-11}$ & CVCoin & $2.2 \times 10^{-10}$ & LGO Exchange & $1.21 \times 10^{-9}$ \\
\hline Filecoin [Fut] & $2.01 \times 10^{-12}$ & Namecoin & $4.17 \times 10^{-11}$ & Olympus Labs & $2.22 \times 10^{-10}$ & Dent & $1.22 \times 10^{-9}$ \\
\hline Eidoo & $2.12 \times 10^{-12}$ & LUXCoin & $4.21 \times 10^{-11}$ & Miners' Rewar & $2.25 \times 10^{-10}$ & Galactrum & $1.22 \times 10^{-9}$ \\
\hline Aeron & $2.19 \times 10^{-12}$ & LATOKEN & $4.21 \times 10^{-11}$ & BitSend & $2.3 \times 10^{-10}$ & ClearPoll & $1.27 \times 10^{-9}$ \\
\hline Chainlink & $2.38 \times 10^{-12}$ & Mercury & $4.25 \times 10^{-11}$ & RefToken & $2.32 \times 10^{-10}$ & $\begin{array}{l}\text { Ethereum } \\
\text { Classic }\end{array}$ & $1.31 \times 10^{-9}$ \\
\hline Arcblock & $2.4 \times 10^{-12}$ & NuBits & $4.28 \times 10^{-11}$ & Speed Mining & $2.34 \times 10^{-10}$ & 1World & $1.31 \times 10^{-9}$ \\
\hline High Performa & $2.52 \times 10^{-12}$ & Dragon Coins & $4.39 \times 10^{-11}$ & Novacoin & $2.36 \times 10^{-10}$ & Cube & $1.33 \times 10^{-9}$ \\
\hline Basic Attenti & $2.68 \times 10^{-12}$ & EDUCare & $4.44 \times 10^{-11}$ & SophiaTX & $2.42 \times 10^{-10}$ & Happycoin & $1.34 \times 10^{-9}$ \\
\hline $\begin{array}{l}\text { Waves } \\
\text { Communi }\end{array}$ & $2.84 \times 10^{-12}$ & BlockMason Cr & $4.46 \times 10^{-11}$ & Covesting & $2.48 \times 10^{-10}$ & SelfSell & $1.36 \times 10^{-9}$ \\
\hline Comet & $2.86 \times 10^{-12}$ & ION & $4.76 \times 10^{-11}$ & ExclusiveCoin & $2.52 \times 10^{-10}$ & DigitalNote & $1.37 \times 10^{-9}$ \\
\hline TenX & $3.03 \times 10^{-12}$ & AppCoins & $4.79 \times 10^{-11}$ & eBoost & $2.56 \times 10^{-10}$ & Bitmark & $1.43 \times 10^{-9}$ \\
\hline PIVX & $3.06 \times 10^{-12}$ & IHT Real Esta & $4.8 \times 10^{-11}$ & Aventus & $2.62 \times 10^{-10}$ & Tokes & $1.44 \times 10^{-9}$ \\
\hline TomoChain & $3.08 \times 10^{-12}$ & Kore & $4.86 \times 10^{-11}$ & CoinMeet & $2.7 \times 10^{-10}$ & BitcoinX & $1.47 \times 10^{-9}$ \\
\hline Melon & $3.15 \times 10^{-12}$ & Matchpool & $4.93 \times 10^{-11}$ & GoldCoin & $2.72 \times 10^{-10}$ & Datawallet & $1.48 \times 10^{-9}$ \\
\hline Zilliqa & $3.42 \times 10^{-12}$ & Nxt & $5.24 \times 10^{-11}$ & Spectre.ai Ut & $2.72 \times 10^{-10}$ & Experty & $1.53 \times 10^{-9}$ \\
\hline Enigma & $3.5 \times 10^{-12}$ & Sphere & $5.35 \times 10^{-11}$ & VeriCoin & $2.72 \times 10^{-10}$ & Indorse Token & $1.54 \times 10^{-9}$ \\
\hline Komodo & $3.7 \times 10^{-12}$ & AirSwap & $5.38 \times 10^{-11}$ & Boolberry & $2.82 \times 10^{-10}$ & More Coin & $1.55 \times 10^{-9}$ \\
\hline SALT & $3.82 \times 10^{-12}$ & Dogecoin & $5.49 \times 10^{-11}$ & Monetha & $2.83 \times 10^{-10}$ & EncrypGen & $1.56 \times 10^{-9}$ \\
\hline Unobtanium & $4.06 \times 10^{-12}$ & Octoin Coin & $5.6 \times 10^{-11}$ & Peerplays & $2.86 \times 10^{-10}$ & EvenCoin & $1.59 \times 10^{-9}$ \\
\hline Aion & $4.5 \times 10^{-12}$ & OctoCoin & $5.6 \times 10^{-11}$ & Pura & $2.96 \times 10^{-10}$ & Wagerr & $1.62 \times 10^{-9}$ \\
\hline Omni & $4.79 \times 10^{-12}$ & Blackmoon & $5.67 \times 10^{-11}$ & Internxt & $3.06 \times 10^{-10}$ & Bitcoin Atom & $1.65 \times 10^{-9}$ \\
\hline Bitcoin Private & $4.89 \times 10^{-12}$ & QLC Chain & $5.94 \times 10^{-11}$ & AidCoin & $3.07 \times 10^{-10}$ & MonetaryUnit & $1.75 \times 10^{-9}$ \\
\hline Primas & $4.95 \times 10^{-12}$ & DECENT & $6.27 \times 10^{-11}$ & TokenClub & $3.08 \times 10^{-10}$ & AMLT & $1.79 \times 10^{-9}$ \\
\hline Gifto & $5.07 \times 10^{-12}$ & Clams & $6.28 \times 10^{-11}$ & LinkEye & $3.15 \times 10^{-10}$ & Diamond & $1.86 \times 10^{-9}$ \\
\hline
\end{tabular}


Table 1. Cont.

\begin{tabular}{|c|c|c|c|c|c|c|c|}
\hline \multicolumn{2}{|c|}{ Group 1 (Most Liquid) } & \multicolumn{2}{|c|}{ Group 2} & \multicolumn{2}{|c|}{ Group 3} & \multicolumn{2}{|c|}{ Group 4} \\
\hline Names & $\begin{array}{l}\text { Amihud } \\
\text { Illiquidity } \\
\text { Ratio }\end{array}$ & Names & $\begin{array}{l}\text { Amihud } \\
\text { Illiquidity } \\
\text { Ratio }\end{array}$ & Names & $\begin{array}{c}\text { Amihud } \\
\text { Illiquidity } \\
\text { Ratio }\end{array}$ & Names & $\begin{array}{l}\text { Amihud } \\
\text { Illiquidity } \\
\text { Ratio }\end{array}$ \\
\hline NEM & $5.17 \times 10^{-12}$ & GridCoin & $6.28 \times 10^{-11}$ & Infinity Econ & $3.15 \times 10^{-10}$ & Kolion & $1.88 \times 10^{-9}$ \\
\hline Aidos Kuneen & $5.2 \times 10^{-12}$ & Edgeless & $6.38 \times 10^{-11}$ & ATBCoin & $3.2 \times 10^{-10}$ & HelloGold & $1.9 \times 10^{-9}$ \\
\hline MonaCoin & $5.25 \times 10^{-12}$ & Viberate & $6.43 \times 10^{-11}$ & UTRUST & $3.22 \times 10^{-10}$ & Sether & $1.95 \times 10^{-9}$ \\
\hline Bitcoin Plus & $5.66 \times 10^{-12}$ & Internet of $\mathrm{P}$ & $6.45 \times 10^{-11}$ & Cindicator & $3.34 \times 10^{-10}$ & Spectrecoin & $1.96 \times 10^{-9}$ \\
\hline
\end{tabular}

Table 2. Groups of less liquid cryptocurrencies.

\begin{tabular}{|c|c|c|c|c|c|c|c|}
\hline \multicolumn{2}{|c|}{ Group 5} & \multicolumn{2}{|c|}{ Group 6} & \multicolumn{2}{|c|}{ Group 7} & \multicolumn{2}{|c|}{ Group 8 (Least Liquid) } \\
\hline Names & $\begin{array}{c}\text { Amihud } \\
\text { Illiquidity } \\
\text { Ratio }\end{array}$ & Names & $\begin{array}{c}\text { Amihud } \\
\text { Illiquidity } \\
\text { Ratio }\end{array}$ & Names & $\begin{array}{c}\text { Amihud } \\
\text { Illiquidity } \\
\text { Ratio }\end{array}$ & Names & $\begin{array}{c}\text { Amihud } \\
\text { Illiquidity } \\
\text { Ratio }\end{array}$ \\
\hline MicroMoney & $1.96 \times 10^{-9}$ & VIVO & $1.46 \times 10^{-8}$ & Neutron & $8.14 \times 10^{-8}$ & Cryptonite & $1.95 \times 10^{-6}$ \\
\hline PRIZM & $1.98 \times 10^{-9}$ & CryptoCarbon & $1.5 \times 10^{-8}$ & HEAT & $8.41 \times 10^{-8}$ & Renos & $2.05 \times 10^{-6}$ \\
\hline Starta & $2 \times 10^{-9}$ & SpaceChain & $1.52 \times 10^{-8}$ & Credo & $8.58 \times 10^{-8}$ & InsaneCoin & $2.05 \times 10^{-6}$ \\
\hline Devery & $2 \times 10^{-9}$ & SwissBorg & $1.53 \times 10^{-8}$ & Tigereum & $9.03 \times 10^{-8}$ & MojoCoin & $2.06 \times 10^{-6}$ \\
\hline Monoeci & $2.01 \times 10^{-9}$ & XTRABYTES & $1.58 \times 10^{-8}$ & AdCoin & $9.15 \times 10^{-8}$ & BlockCDN & $2.16 \times 10^{-6}$ \\
\hline LALA World & $2.09 \times 10^{-9}$ & Sequence & $1.61 \times 10^{-8}$ & Crave & $9.17 \times 10^{-8}$ & TOKYO & $2.21 \times 10^{-6}$ \\
\hline Chronologic & $2.1 \times 10^{-9}$ & BitClave & $1.62 \times 10^{-8}$ & MarteXcoin & $9.35 \times 10^{-8}$ & GoldBlocks & $2.34 \times 10^{-6}$ \\
\hline WeTrust & $2.1 \times 10^{-9}$ & Nexium & $1.64 \times 10^{-8}$ & PeepCoin & $9.67 \times 10^{-8}$ & Bitcoin Red & $2.46 \times 10^{-6}$ \\
\hline XPA & $2.16 \times 10^{-9}$ & Bela & $1.65 \times 10^{-8}$ & BlitzPredict & $9.9 \times 10^{-8}$ & Universe & $2.51 \times 10^{-6}$ \\
\hline Endor Protocol & $2.21 \times 10^{-9}$ & OFCOIN & $1.74 \times 10^{-8}$ & SoMee.Social & $1.01 \times 10^{-7}$ & ELTCOIN & $2.55 \times 10^{-6}$ \\
\hline OracleChain & $2.22 \times 10^{-9}$ & DomRaider & $1.75 \times 10^{-8}$ & Orbitcoin & $1.05 \times 10^{-7}$ & LiteCoin Ultra & $2.83 \times 10^{-6}$ \\
\hline PotCoin & $2.25 \times 10^{-9}$ & CannaCoin & $1.82 \times 10^{-8}$ & Authorship & $1.05 \times 10^{-7}$ & LIFE & $2.87 \times 10^{-6}$ \\
\hline SpankChain & $2.29 \times 10^{-9}$ & SHIELD & $1.84 \times 10^{-8}$ & BitDegree & $1.08 \times 10^{-7}$ & Ratecoin & $2.98 \times 10^{-6}$ \\
\hline Maverick Chain & $2.33 \times 10^{-9}$ & BitStation & $1.85 \times 10^{-8}$ & MktCoin & $1.12 \times 10^{-7}$ & Version & $3.17 \times 10^{-6}$ \\
\hline BitBar & $2.35 \times 10^{-9}$ & OBITS & $1.89 \times 10^{-8}$ & Dentacoin & $1.17 \times 10^{-7}$ & BitCoal & $3.69 \times 10^{-6}$ \\
\hline Auroracoin & $2.46 \times 10^{-9}$ & Debitum & $1.91 \times 10^{-8}$ & Deutsche eMark & $1.18 \times 10^{-7}$ & PostCoin & $3.7 \times 10^{-6}$ \\
\hline Pillar & $2.47 \times 10^{-9}$ & Cryptopay & $1.93 \times 10^{-8}$ & DigitalPrice & $1.19 \times 10^{-7}$ & BiblePay & $3.76 \times 10^{-6}$ \\
\hline Ignition & $2.48 \times 10^{-9}$ & Graft & $1.99 \times 10^{-8}$ & Storjcoin X & $1.21 \times 10^{-7}$ & AudioCoin & $3.92 \times 10^{-6}$ \\
\hline Cashaa & $2.49 \times 10^{-9}$ & Payfair & $2.01 \times 10^{-8}$ & PetroDollar & $1.23 \times 10^{-7}$ & Joulecoin & $4.13 \times 10^{-6}$ \\
\hline HOQU & $2.56 \times 10^{-9}$ & EDRCoin & $2.02 \times 10^{-8}$ & Pesetacoin & $1.24 \times 10^{-7}$ & Fujinto & $4.37 \times 10^{-6}$ \\
\hline Universal Cur... & $2.57 \times 10^{-9}$ & Sociall & $2.07 \times 10^{-8}$ & Vezt & $1.24 \times 10^{-7}$ & BlueCoin & $4.58 \times 10^{-6}$ \\
\hline Veros & $2.67 \times 10^{-9}$ & Qube & $2.08 \times 10^{-8}$ & Circuits of V... & $1.28 \times 10^{-7}$ & Blue Protocol & $4.58 \times 10^{-6}$ \\
\hline Datum & $2.67 \times 10^{-9}$ & WhaleCoin & $2.13 \times 10^{-8}$ & Bata & $1.37 \times 10^{-7}$ & XRP & $4.78 \times 10^{-6}$ \\
\hline Energo & $2.67 \times 10^{-9}$ & EverGreenCoin & $2.14 \times 10^{-8}$ & carVertical & $1.48 \times 10^{-7}$ & Atomic Coin & $5.06 \times 10^{-6}$ \\
\hline Hush & $2.7 \times 10^{-9}$ & Engine & $2.14 \times 10^{-8}$ & InvestFeed & $1.54 \times 10^{-7}$ & Swisscoin & $5.25 \times 10^{-6}$ \\
\hline PIECoin & $2.72 \times 10^{-9}$ & Zap & $2.14 \times 10^{-8}$ & Flash & $1.57 \times 10^{-7}$ & HiCoin & $5.53 \times 10^{-6}$ \\
\hline $\begin{array}{l}\text { MediBloc } \\
\text { [QRC20] }\end{array}$ & $2.75 \times 10^{-9}$ & Innova & $2.19 \times 10^{-8}$ & Rise & $1.57 \times 10^{-7}$ & Digital Money & $5.84 \times 10^{-6}$ \\
\hline Litecoin Cash & $2.81 \times 10^{-9}$ & Dovu & $2.34 \times 10^{-8}$ & ATMChain & $1.6 \times 10^{-7}$ & HyperStake & $5.91 \times 10^{-6}$ \\
\hline Databits & $2.86 \times 10^{-9}$ & Gold Bits Coin & $2.37 \times 10^{-8}$ & iEthereum & $1.68 \times 10^{-7}$ & HyperStake & $5.91 \times 10^{-6}$ \\
\hline Gulden & $3.01 \times 10^{-9}$ & LOCIcoin & $2.38 \times 10^{-8}$ & Opus & $1.71 \times 10^{-7}$ & SecureCoin & $5.97 \times 10^{-6}$ \\
\hline AICHAIN & $3.03 \times 10^{-9}$ & Dynamic Tradi... & $2.39 \times 10^{-8}$ & 2GIVE & $1.81 \times 10^{-7}$ & Superior Coin & $6.45 \times 10^{-6}$ \\
\hline HempCoin & $3.06 \times 10^{-9}$ & bitJob & $2.42 \times 10^{-8}$ & ArtByte & $1.93 \times 10^{-7}$ & TajCoin & $6.56 \times 10^{-6}$ \\
\hline Gladius Token & $3.15 \times 10^{-9}$ & CFun & $2.43 \times 10^{-8}$ & Kin & $1.93 \times 10^{-7}$ & XGOX & $7.04 \times 10^{-6}$ \\
\hline Content and A... & $3.17 \times 10^{-9}$ & Minereum & $2.43 \times 10^{-8}$ & Bitcloud & $1.99 \times 10^{-7}$ & Rimbit & $7.4 \times 10^{-6}$ \\
\hline SpreadCoin & $3.17 \times 10^{-9}$ & Safe Exchange & $2.46 \times 10^{-8}$ & Sparks & $2.06 \times 10^{-7}$ & Eternity & $7.41 \times 10^{-6}$ \\
\hline Rubycoin & $3.25 \times 10^{-9}$ & DraftCoin & $2.48 \times 10^{-8}$ & Intelligent T & $2.07 \times 10^{-7}$ & FujiCoin & $8.01 \times 10^{-6}$ \\
\hline OceanChain & $3.45 \times 10^{-9}$ & Pepe Cash & $2.62 \times 10^{-8}$ & $\mathrm{Cl}$ & $2.08 \times 10^{-7}$ & Xenon & $8.04 \times 10^{-6}$ \\
\hline VouchForMe & $3.69 \times 10^{-9}$ & COPYTRACK & $2.63 \times 10^{-8}$ & Zetacoin & $2.13 \times 10^{-7}$ & Pakcoin & $8.24 \times 10^{-6}$ \\
\hline PiplCoin & $3.8 \times 10^{-9}$ & MaxCoin & $2.67 \times 10^{-8}$ & ZetaMicron & $2.13 \times 10^{-7}$ & Eryllium & $8.54 \times 10^{-6}$ \\
\hline CoinPoker & $3.84 \times 10^{-9}$ & Snovian.Space & $2.7 \times 10^{-8}$ & Advanced Tech & $2.14 \times 10^{-7}$ & MintCoin & $9.36 \times 10^{-6}$ \\
\hline
\end{tabular}


Table 2. Cont.

\begin{tabular}{|c|c|c|c|c|c|c|c|}
\hline \multicolumn{2}{|c|}{ Group 5} & \multicolumn{2}{|c|}{ Group 6} & \multicolumn{2}{|c|}{ Group 7} & \multicolumn{2}{|c|}{ Group 8 (Least Liquid) } \\
\hline Names & $\begin{array}{c}\text { Amihud } \\
\text { Illiquidity } \\
\text { Ratio }\end{array}$ & Names & $\begin{array}{c}\text { Amihud } \\
\text { Illiquidity } \\
\text { Ratio }\end{array}$ & Names & $\begin{array}{c}\text { Amihud } \\
\text { Illiquidity } \\
\text { Ratio }\end{array}$ & Names & $\begin{array}{c}\text { Amihud } \\
\text { Illiquidity } \\
\text { Ratio }\end{array}$ \\
\hline Universa & $3.95 \times 10^{-9}$ & Golos & $2.75 \times 10^{-8}$ & Unify & $2.15 \times 10^{-7}$ & Profile Utili... & $9.58 \times 10^{-6}$ \\
\hline Bonpay & $3.96 \times 10^{-9}$ & Social Send & $2.92 \times 10^{-8}$ & The ChampCoin & $2.2 \times 10^{-7}$ & Helleniccoin & $9.93 \times 10^{-6}$ \\
\hline Gems & $4.06 \times 10^{-9}$ & LoMoCoin & $2.93 \times 10^{-8}$ & Ixcoin & $2.26 \times 10^{-7}$ & Z & $1 \times 10^{-5}$ \\
\hline Incent & $4.16 \times 10^{-9}$ & GeyserCoin & $2.93 \times 10^{-8}$ & Ixcoin & $2.26 \times 10^{-7}$ & Nekonium & $1.03 \times 10^{-5}$ \\
\hline Bitcrystals & $4.21 \times 10^{-9}$ & Yocoin & $3 \times 10^{-8}$ & Mao Zedong & $2.49 \times 10^{-7}$ & First Bitcoin & $1.04 \times 10^{-5}$ \\
\hline BitRent & $4.42 \times 10^{-9}$ & Crystal Clear & $3.06 \times 10^{-8}$ & OP Coin & $2.63 \times 10^{-7}$ & First Bitcoin & $1.04 \times 10^{-5}$ \\
\hline Verify & $4.42 \times 10^{-9}$ & SounDAC & $3.07 \times 10^{-8}$ & $\begin{array}{l}\text { UNIVERSAL } \\
\text { CASH }\end{array}$ & $2.64 \times 10^{-7}$ & BumbaCoin & $1.05 \times 10^{-5}$ \\
\hline Zero & $4.49 \times 10^{-9}$ & Viuly & $3.1 \times 10^{-8}$ & BitcoinZ & $2.67 \times 10^{-7}$ & LiteBitcoin & $1.08 \times 10^{-5}$ \\
\hline Stealth & $4.51 \times 10^{-9}$ & Terracoin & $3.14 \times 10^{-8}$ & TeslaCoin & $2.85 \times 10^{-7}$ & BigUp & $1.12 \times 10^{-5}$ \\
\hline Peculium & $4.96 \times 10^{-9}$ & Sugar Exchange & $3.16 \times 10^{-8}$ & TeslaCoin & $2.85 \times 10^{-7}$ & FedoraCoin & $1.16 \times 10^{-5}$ \\
\hline ShipChain & $4.96 \times 10^{-9}$ & FirstCoin & $3.18 \times 10^{-8}$ & NEVERDIE & $2.91 \times 10^{-7}$ & Bitcoin Planet & $1.2 \times 10^{-5}$ \\
\hline Breakout Stake & $5.03 \times 10^{-9}$ & Dropil & $3.2 \times 10^{-8}$ & Ccore & $2.91 \times 10^{-7}$ & Titcoin & $1.25 \times 10^{-5}$ \\
\hline Karbo & $5.1 \times 10^{-9}$ & FlypMe & $3.21 \times 10^{-8}$ & ToaCoin & $2.98 \times 10^{-7}$ & CoinonatX & $1.44 \times 10^{-5}$ \\
\hline Soma & $5.33 \times 10^{-9}$ & PlatinumBAR & $3.27 \times 10^{-8}$ & EA Coin & $3.29 \times 10^{-7}$ & Phantomx & $1.67 \times 10^{-5}$ \\
\hline TE-FOOD & $5.42 \times 10^{-9}$ & HunterCoin & $3.4 \times 10^{-8}$ & Mincoin & $3.32 \times 10^{-7}$ & PopularCoin & $1.67 \times 10^{-5}$ \\
\hline Education Eco & $5.45 \times 10^{-9}$ & HunterCoin & $3.4 \times 10^{-8}$ & Linker Coin & $3.49 \times 10^{-7}$ & Luna Coin & $1.75 \times 10^{-5}$ \\
\hline Block Array & $5.53 \times 10^{-9}$ & Litecoin Plus & $3.43 \times 10^{-8}$ & Blocklancer & $3.49 \times 10^{-7}$ & bitqy & $1.94 \times 10^{-5}$ \\
\hline Curecoin & $5.6 \times 10^{-9}$ & Bee Token & $3.45 \times 10^{-8}$ & Safecoin & $3.49 \times 10^{-7}$ & Coinonat & $2 \times 10^{-5}$ \\
\hline aXpire & $5.77 \times 10^{-9}$ & indaHash & $3.47 \times 10^{-8}$ & Dalecoin & $3.92 \times 10^{-7}$ & Goodomy & $2.05 \times 10^{-5}$ \\
\hline REAL & $5.82 \times 10^{-9}$ & DopeCoin & $3.6 \times 10^{-8}$ & Moin & $4 \times 10^{-7}$ & Pandacoin & $2.11 \times 10^{-5}$ \\
\hline CoinFi & $5.84 \times 10^{-9}$ & Voise & $3.71 \times 10^{-8}$ & Honey & $4.19 \times 10^{-7}$ & BuzzCoin & $2.13 \times 10^{-5}$ \\
\hline Banca & $5.91 \times 10^{-9}$ & Cobinhood & $3.77 \times 10^{-8}$ & Motocoin & $4.7 \times 10^{-7}$ & SmartCoin & $2.41 \times 10^{-5}$ \\
\hline HEROcoin & $5.91 \times 10^{-9}$ & PinkCoin & $3.79 \times 10^{-8}$ & Elcoin & $4.74 \times 10^{-7}$ & Bitbase & $2.49 \times 10^{-5}$ \\
\hline Sovereign Hero & $5.91 \times 10^{-9}$ & Rupee & $3.84 \times 10^{-8}$ & Equal & $4.82 \times 10^{-7}$ & Argus & $2.64 \times 10^{-5}$ \\
\hline Show & $5.97 \times 10^{-9}$ & BitDice & $3.93 \times 10^{-8}$ & Garlicoin & $4.89 \times 10^{-7}$ & Dinastycoin & $2.66 \times 10^{-5}$ \\
\hline SolarCoin & $6.04 \times 10^{-9}$ & MaxCoin & $4.09 \times 10^{-8}$ & BTCMoon & $4.95 \times 10^{-7}$ & AnarchistsPrime & $3.21 \times 10^{-5}$ \\
\hline Rivetz & $6.05 \times 10^{-9}$ & SkinCoin & $4.2 \times 10^{-8}$ & BioCoin & $5.15 \times 10^{-7}$ & Cryptojacks & $3.29 \times 10^{-5}$ \\
\hline AdShares & $6.12 \times 10^{-9}$ & GridCoin & $4.29 \times 10^{-8}$ & Bitradio & $5.61 \times 10^{-7}$ & NewYorkCoin & $3.33 \times 10^{-5}$ \\
\hline BitClave & $6.2 \times 10^{-9}$ & Tracto & $4.38 \times 10^{-8}$ & GlobalBoost-Y & $5.72 \times 10^{-7}$ & YENTEN & $3.51 \times 10^{-5}$ \\
\hline Zeusshield & $6.24 \times 10^{-9}$ & DAO.Casino & $4.41 \times 10^{-8}$ & High Voltage & $5.72 \times 10^{-7}$ & AmsterdamCoin & $3.96 \times 10^{-5}$ \\
\hline SegWit $2 x$ & $6.32 \times 10^{-9}$ & Animation Vis & $4.72 \times 10^{-8}$ & ZenGold & $5.78 \times 10^{-7}$ & LiteDoge & $4.06 \times 10^{-5}$ \\
\hline Cappasity & $6.5 \times 10^{-9}$ & MedicCoin & $4.76 \times 10^{-8}$ & HTMLCOIN & $6.07 \times 10^{-7}$ & Uniform Fisca & $4.15 \times 10^{-5}$ \\
\hline MCAP & $6.65 \times 10^{-9}$ & Eroscoin & $5.18 \times 10^{-8}$ & Skeincoin & $6.22 \times 10^{-7}$ & Trollcoin & $4.42 \times 10^{-5}$ \\
\hline Global Crypto & $6.81 \times 10^{-9}$ & EventChain & $5.32 \times 10^{-8}$ & Comet & $6.31 \times 10^{-7}$ & Dimecoin & $4.97 \times 10^{-5}$ \\
\hline SpeedCash & $6.91 \times 10^{-9}$ & TrumpCoin & $5.39 \times 10^{-8}$ & TrezarCoin & $6.33 \times 10^{-7}$ & BERNcash & $5.04 \times 10^{-5}$ \\
\hline Elixir & $6.93 \times 10^{-9}$ & Telcoin & $5.46 \times 10^{-8}$ & Oceanlab & $6.52 \times 10^{-7}$ & Coupecoin & $5.1 \times 10^{-5}$ \\
\hline Maecenas & $7.27 \times 10^{-9}$ & Spectiv & $5.47 \times 10^{-8}$ & Regalcoin & $6.75 \times 10^{-7}$ & Grimcoin & $5.1 \times 10^{-5}$ \\
\hline Ink Protocol & $7.82 \times 10^{-9}$ & ALQO & $5.51 \times 10^{-8}$ & $\begin{array}{l}\text { President } \\
\text { Trump }\end{array}$ & $7.14 \times 10^{-7}$ & $\begin{array}{c}\text { Theresa May } \\
\text { Coin }\end{array}$ & $5.75 \times 10^{-5}$ \\
\hline Blue Protocol & $8.11 \times 10^{-9}$ & ColossusXT & $5.51 \times 10^{-8}$ & Quark & $7.27 \times 10^{-7}$ & Nyancoin & $5.92 \times 10^{-5}$ \\
\hline Alphacat & $8.18 \times 10^{-9}$ & PACcoin & $5.57 \times 10^{-8}$ & Onix & $7.56 \times 10^{-7}$ & Blakecoin & $5.92 \times 10^{-5}$ \\
\hline Xaurum & $8.39 \times 10^{-9}$ & Argentum & $5.63 \times 10^{-8}$ & Digitalcoin & $7.58 \times 10^{-7}$ & Megacoin & $5.92 \times 10^{-5}$ \\
\hline Bean Cash & $8.6 \times 10^{-9}$ & Desire & $5.65 \times 10^{-8}$ & Ethereum Cash & $7.6 \times 10^{-7}$ & Elite & $5.93 \times 10^{-5}$ \\
\hline Pareto Network & $8.77 \times 10^{-9}$ & Obsidian & $5.72 \times 10^{-8}$ & FORCE & $7.62 \times 10^{-7}$ & C-Bit & $6.1 \times 10^{-5}$ \\
\hline Bounty0x & $9.02 \times 10^{-9}$ & Soarcoin & $5.72 \times 10^{-8}$ & Adzcoin & $8.24 \times 10^{-7}$ & TEKcoin & $8.39 \times 10^{-5}$ \\
\hline IP Exchange & $9.85 \times 10^{-9}$ & Billionaire T... & $5.86 \times 10^{-8}$ & Francs & $9.05 \times 10^{-7}$ & Zeitcoin & $9.66 \times 10^{-5}$ \\
\hline Dether & $9.91 \times 10^{-9}$ & X-Coin & $5.89 \times 10^{-8}$ & WavesGo & $9.24 \times 10^{-7}$ & ZEIT & $9.66 \times 10^{-5}$ \\
\hline Sharpe Platfo.. & $9.94 \times 10^{-9}$ & Musicoin & $5.91 \times 10^{-8}$ & Lendroid Supp & $9.26 \times 10^{-7}$ & HOdlcoin & $9.81 \times 10^{-5}$ \\
\hline SiaCashCoin & $1.01 \times 10^{-8}$ & EquiTrader & $5.95 \times 10^{-8}$ & VoteCoin & $1.01 \times 10^{-6}$ & Piggycoin & $1.03 \times 10^{-4}$ \\
\hline MyWish & $1.06 \times 10^{-8}$ & $\begin{array}{l}\text { Dinnerful } \\
\text { Network }\end{array}$ & $6.05 \times 10^{-8}$ & ArbitrageCT & $1.01 \times 10^{-6}$ & SongCoin & $1.07 \times 10^{-4}$ \\
\hline ATLANT & $1.11 \times 10^{-8}$ & FoldingCoin & $6.1 \times 10^{-8}$ & ERA & $1.01 \times 10^{-6}$ & Golfcoin & $1.18 \times 10^{-4}$ \\
\hline BlockCDN & $1.12 \times 10^{-8}$ & ParallelCoin & $6.1 \times 10^{-8}$ & Sharechain & $1.04 \times 10^{-6}$ & Jesus Coin & $1.44 \times 10^{-4}$ \\
\hline Autonio & $1.14 \times 10^{-8}$ & Ellaism & $6.14 \times 10^{-8}$ & Swing & $1.08 \times 10^{-6}$ & DynamicCoin & $1.48 \times 10^{-4}$ \\
\hline Read & $1.15 \times 10^{-8}$ & StarterCoin & $6.18 \times 10^{-8}$ & Cream & $1.16 \times 10^{-6}$ & POLY AI & $1.62 \times 10^{-4}$ \\
\hline FairCoin & $1.15 \times 10^{-8}$ & Fluz Fluz & $6.53 \times 10^{-8}$ & Bitdeal & $1.16 \times 10^{-6}$ & CrevaCoin & $2.49 \times 10^{-4}$ \\
\hline
\end{tabular}


Table 2. Cont.

\begin{tabular}{cccccccc}
\hline \multicolumn{2}{c}{ Group 5 } & \multicolumn{2}{c}{ Group 6 } & \multicolumn{2}{c}{ Group 7 } & \multicolumn{2}{c}{ Group 8 (Least Liquid) } \\
\hline Names & $\begin{array}{c}\text { Amihud } \\
\text { Illiquidity } \\
\text { Ratio }\end{array}$ & Names & $\begin{array}{c}\text { Amihud } \\
\text { Illiquidity } \\
\text { Ratio }\end{array}$ & Names & $\begin{array}{c}\text { Amihud } \\
\text { Illiquidity } \\
\text { Ratio }\end{array}$ & $\begin{array}{c}\text { Amihud } \\
\text { Names } \\
\text { Illiquidity } \\
\text { Ratio }\end{array}$ \\
\hline Tidex Token & $1.16 \times 10^{-8}$ & Tokenbox & $6.62 \times 10^{-8}$ & Centurion & $1.31 \times 10^{-6}$ & BunnyCoin & $5.01 \times 10^{-4}$ \\
\hline STRAKS & $1.18 \times 10^{-8}$ & AquariusCoin & $6.63 \times 10^{-8}$ & Startcoin & $1.35 \times 10^{-6}$ & Tellurion & $6.64 \times 10^{-4}$ \\
\hline Breakout & $1.21 \times 10^{-8}$ & DIMCOIN & $6.7 \times 10^{-8}$ & Master Swiscoin & $1.44 \times 10^{-6}$ & Carboncoin & $8.57 \times 10^{-4}$ \\
\hline eBitcoin & $1.27 \times 10^{-8}$ & Qbic & $6.73 \times 10^{-8}$ & Bolivarcoin & $1.58 \times 10^{-6}$ & InflationCoin & $8.89 \times 10^{-4}$ \\
\hline Travelflex & $1.29 \times 10^{-8}$ & Myriad & $7.08 \times 10^{-8}$ & Ethereum Gold & $1.59 \times 10^{-6}$ & FunFair & $1.06 \times 10^{-3}$ \\
\hline ALIS & $1.33 \times 10^{-8}$ & Pure & $7.09 \times 10^{-8}$ & WorldCoin & $1.61 \times 10^{-6}$ & StrongHands & $1.27 \times 10^{-3}$ \\
\hline e-Gulden & $1.33 \times 10^{-8}$ & PlanetPay & $7.1 \times 10^{-8}$ & ECC & $1.61 \times 10^{-6}$ & 808 Coin & $1.61 \times 10^{-3}$ \\
\hline DCORP Utility & $1.37 \times 10^{-8}$ & JET8 & $7.55 \times 10^{-8}$ & Condensate & $1.64 \times 10^{-6}$ & Dix Asset & $3.58 \times 10^{-3}$ \\
\hline FidentiaX & $1.37 \times 10^{-8}$ & HomeBlockCoin & $7.73 \times 10^{-8}$ & Shekel & $1.65 \times 10^{-6}$ & Sprouts & $7.08 \times 10^{-3}$ \\
\hline Jetcoin & $1.39 \times 10^{-8}$ & Elementrem & $7.9 \times 10^{-8}$ & HarmonyCoin & $1.78 \times 10^{-6}$ & WeAreSatoshi & $1.73 \times 10^{-2}$ \\
\hline NoLimitCoin & $1.43 \times 10^{-8}$ & Lampix & $7.98 \times 10^{-8}$ & Experience Po... & $1.85 \times 10^{-6}$ & \\
\hline Magi & $1.44 \times 10^{-8}$ & adbank & $8.14 \times 10^{-8}$ & NevaCoin & $1.87 \times 10^{-6}$ & \\
\hline
\end{tabular}

It can be observed that the most important coins in the cryptocurrency markets, such as Bitcoin, Ethereum, Monero, BitcoinCash, IOTA, Tether, EOS, Ethereum Classic, Stellar, Stratis, Dash and Cardano remain the most popular across investor trading preferences even in bearish periods, despite their large price decreases. In this highest liquidity group, the Amihud's illiquidity ratio takes values from $9.09 \times 10^{-28}$ (most liquid) to $5.66 \times 10^{-12}$ (least liquid in this group). The number of active traders of these currencies remains high as it costs less for investors and speculators to trade with these coins due to lower spreads as well as lower transaction costs. Investors believe that if the liquidity of a cryptocurrency is low, it is more difficult to make profit from it as it would be more time-consuming to find a preference matching with other traders willing to make the opposite move. This is why more liquid assets are found to be more preferable during distressed times and not only during flourishing periods that previous academic work has shown (Wei 2018).

On the other hand, less known virtual currencies such as the 808, Dix Asset, Sprouts and WeAreSatoshi appear to exhibit much lower levels of liquidity. More specifically, the Amihud's illiquidity ratio takes values from $1.95 \times 10^{-6}$ to 0.017296 in this eighth group of currencies that stands for the least liquid digital coins. This provides evidence that investors do not prefer during bearish periods currencies that traditionally exhibit low market capitalization, as they are considered more costly to invest in.

Overall, our findings are partly in line with conventional methods for measuring liquidity, based on, such as, the market capitalization or the trading volume of digital currencies. As one can easily observe, the great majority of high-capitalization cryptocurrencies are found to be the most liquid ones according to the Amihud's illiquidity ratio. Moreover, lower-capitalization currencies are part of the lower-liquidity groups based on the categorization performed by Amihud's method. Liquidity examination based on trading volume brings about similar outcomes.

The results of our study are informative about the potential that investors have on substituting investments in high-capitalization cryptocurrencies with lower-capitalization ones during turbulent times when market values of Bitcoin are decreasing. Evidence reveals that there is little tendency of economic agents to invest in alternative digital currencies, but only in the best-known ones. It is remarkable that the most liquid currency during this bearish period, which leaves Bitcoin at the second place, has been the TrueUSD stablecoin that is tied to the US dollar. This is very informative about investors' preferences during distressed times. Notably, they do not abandon investments in the already unstable cryptocurrencies in order to invest in less known and probably more volatile ones, but they rather invest in a coin tied to the most prestigious international currency that carries a global legal tender. These findings reveal that economic units tend to diversify their portfolios and hold a mildly more risk-averse investment profile during crises rather than a more risky attitude. 


\section{Conclusions}

Liquidity has been one of the principal interests of investors and speculators when they decide how to conduct active trading on a particular asset. Cryptocurrencies constitute innovative forms of investment assets where markets are characterized by high levels of herding behavior and a very large portion of market capitalization is concentrated in a small number of important currencies.

This study undertakes the task of estimating the level of liquidity during bearish times in cryptocurrency markets for every digital currency on about which full data are provided in a daily frequency. Thereby, the extra-bearish second phase of downwards movements in digital currency markets is under scrutiny, spanning from 1 April 2018-when the second very sudden drop in digital coin prices took place - until 31 January 2019 when the market started recovering. This paper casts light on whether the active trading preferences of investors are more favorable for well-established cryptocurrencies such as Bitcoin, Ethereum, Ripple, Litecoin, BitcoinCash, Cardano, Stellar and Nem. Previous academic work (Bouri et al. 2019a) has identified these currencies as the primary determinants of herding behavior.

The Amihud's illiquidity ratio by Amihud (2002) enables us to categorize the finally short-listed digital currencies into eight groups, where the first group stands for lower values of the ratio, that is the most liquid cryptocurrencies. Results reveal that the leading virtual currencies that also exhibit the highest market capitalization in normal times, remain the most actively traded digital assets also during bearish periods. The Amihud's illiquidity ratio takes values from $9.09 \times 10^{-28}$ (most liquid) to $5.66 \times 10^{-12}$ (least liquid) in this first group that presents the highest levels of liquidity. On the other hand, the eighth (least liquid) group comprises of significantly less known virtual coins that are much more costly and time-consuming to invest in. This group of least liquid currencies present values for Amihud's illiquidity ratio that are significantly lower than those of previous groups. Lower spreads and lower transaction costs remain the basic determinants of popularity that most liquid cryptocurrencies enjoy, even in distressed times. It is worth noting that the highest-capitalization digital currencies remain the most liquid during distressed periods. Intriguingly, the most liquid currency is found to be the TrueUSD stablecoin which is tied to the US dollar. This reveals that during bearish trends in the cryptocurrency market, investors are slightly more adverse to risk. This is the reason why they diversify their portfolios through the inclusion of less volatile currencies tied to legal and widely-approved forms of money.

This paper is the first one to provide an overall view of the liquidity levels concerning a very wide range of digital currencies during the intensely bearish period in digital currency investments. The main research axis of this study lies on investigating how the popularity and the incumbent character of specific coins in the virtual currency markets affect decision-making about active trading. Our results reinforce previous findings that support the existence of a leading group of high-capitalization currencies being the determinants of herding behavior and the vivid investor sentiment in cryptocurrency markets.

Author Contributions: Conceptualisation and methodology, N.A.K.; software and validation, P.P.; investigation, N.A.K. and P.P.; writing-original draft preparation, N.A.K. and P.P.; writing-review and editing, N.A.K. and P.P.; and supervision, N.A.K.

Funding: This research received no external funding.

Conflicts of Interest: The authors declare no conflict of interest.

\section{References}

Adhami, Saman, Giancarlo Giudici, and Stefano Martinazzi. 2018. Why do businesses go crypto? An empirical analysis of initial coin offerings. Journal of Economics and Business 100: 64-75. [CrossRef]

Amihud, Yakov. 2002. Illiquidity and stock returns: cross-section and time-series effects. Journal of Financial Markets 5: 31-56. [CrossRef]

Ammous, Saifedean. 2018. Can cryptocurrencies fulfil the functions of money? The Quarterly Review of Economics and Finance 70: 38-51. [CrossRef] 
Balcilar, Mehmet, Elie Bouri, Rangan Gupta, and David Roubaud. 2017. Can volume predict Bitcoin returns and volatility? A quantiles-based approach. Economic Modelling 64: 74-81. [CrossRef]

Baur, Dirk G., KiHoon Hong, and Adrian D. Lee. 2018. Bitcoin: Medium of exchange or speculative assets? Journal of International Financial Markets, Institutions and Money 54: 177-89. [CrossRef]

Beneki, Christina, Alexandros Koulis, Nikolaos A. Kyriazis, and Stephanos Papadamou. 2019. Investigating Volatility Transmission and Hedging Properties between Bitcoin and Ethereum. Research in International Business and Finance 48: 219-27. [CrossRef]

Bouri, Elie, Rangan Gupta, Aviral Kumar Tiwari, and David Roubaud. 2017. Does Bitcoin hedge global uncertainty? Evidence from wavelet-based quantile-in-quantile regressions. Finance Research Letters 23: 87-95. [CrossRef]

Bouri, Elie, Syed Jawad Hussain Shahzad, and David Roubaud. 2018. Co-explosivity in the cryptocurrency market. Finance Research Letters 29: 178-83. [CrossRef]

Bouri, Elie, Luis A. Gil-Alana, Rangan Gupta, and David Roubaud. 2019a. Modelling long memory volatility in the Bitcoin market: Evidence of persistence and structural breaks. International Journal of Finance E Economics 24: 412-26.

Bouri, Elie, Rangan Gupta, and David Roubaud. 2019b. Herding behaviour in cryptocurrencies. Finance Research Letters 29: 216-21. [CrossRef]

Bouri, Elie, Chi Keung Marco Lau, Brian Lucey, and David Roubaud. 2019c. Trading volume and the predictability of return and volatility in the cryptocurrency market. Finance Research Letters 29: 340-46. [CrossRef]

Brandvold, Morten, Peter Molnár, Kristian Vagstad, and Ole Christian Andreas Valstad. 2015. Price discovery on Bitcoin exchanges. Journal of International Financial Markets, Institutions and Money 36: 18-35. [CrossRef]

Corbet, Shaen, Andrew Meegan, Charles Larkin, Brian Lucey, and Larisa Yarovaya. 2018. Exploring the dynamic relationships between cryptocurrencies and other financial assets. Economics Letters 165: 28-34. [CrossRef]

Corbet, Shaen, Brian Lucey, Andrew Urquhart, and Larisa Yarovaya. 2019. Cryptocurrencies as a financial asset: A systematic analysis. International Review of Financial Analysis 62: 182-99. [CrossRef]

Dastgir, Shabbir, Ender Demir, Gareth Downing, Giray Gozgor, and Chi Keung Marco Lau. 2019. The causal relationship between Bitcoin attention and Bitcoin returns: Evidence from the Copula-based Granger causality test. Finance Research Letters 28: 160-64. [CrossRef]

Dyhrberg, Anne Haubo. 2016a. Bitcoin, gold and the dollar-A GARCH volatility analysis. Finance Research Letters 16: 85-92. [CrossRef]

Dyhrberg, Anne Haubo. 2016b. Hedging capabilities of bitcoin. Is it the virtual gold? Finance Research Letters 16: 139-44. [CrossRef]

Feng, Wenjun, Yiming Wang, and Zhengjun Zhang. 2018. Informed trading in the Bitcoin market. Finance Research Letters 26: 63-70. [CrossRef]

Ferreira, Paulo, and Éder Pereira. 2019. Contagion Effect in Cryptocurrency Market. Journal of Risk and Financial Management 12: 115. [CrossRef]

Gandal, Neil, J. T. Hamrick, Tyler Moore, and Tali Oberman. 2018. Price manipulation in the Bitcoin ecosystem. Journal of Monetary Economics 95: 86-96. [CrossRef]

Giudici, Paolo, and Iman Abu-Hashish. 2019. What determines bitcoin exchange prices? A network VAR approach. Finance Research Letters 28: 309-18. [CrossRef]

Hayes, Adam S. 2017. Cryptocurrency value formation: An empirical study leading to a cost of production model for valuing bitcoin. Telematics and Informatics 34: 1308-21. [CrossRef]

Hyun, Steve, Jimin Lee, Jong-Min Kim, and Chulhee Jun. 2019. What Coins Lead in the Cryptocurrency Market: Using Copula and Neural Networks Models. Journal of Risk and Financial Management 12: 132. [CrossRef]

Ji, Qiang, Elie Bouri, Chi Keung Marco Lau, and David Roubaud. 2019. Dynamic connectedness and integration in cryptocurrency markets. International Review of Financial Analysis 63: 257-72. [CrossRef]

Katsiampa, Paraskevi. 2017. Volatility estimation for Bitcoin: A comparison of GARCH models. Economics Letters 158: 3-6. [CrossRef]

Kim, Thomas. 2017. On the transaction cost of Bitcoin. Finance Research Letters 23: 300-5. [CrossRef]

Kyriazis, Nikolaos A. 2019. A Survey on Efficiency and Profitable Trading Opportunities in Cryptocurrency Markets. Journal of Risk and Financial Management 12: 67. [CrossRef]

Kyriazis, Nikolaos A., Kalliopi Daskalou, Marios Arampatzis, Paraskevi Prassa, and Evangelia Papaioannou. 2019. Estimating the volatility of cryptocurrencies during bearish markets by employing GARCH models. Heliyon 5: e02239. [CrossRef] 
Panagiotidis, Theodore, Thanasis Stengos, and Orestis Vravosinos. 2018. On the determinants of bitcoin returns: A LASSO approach. Finance Research Letters 27: 235-40. [CrossRef]

Pieters, Gina, and Sofia Vivanco. 2017. Financial regulations and price inconsistencies across Bitcoin markets. Information Economics and Policy 39: 1-14. [CrossRef]

Schilling, Linda, and Harald Uhlig. 2019. Some simple bitcoin economics. Journal of Monetary Economics. [CrossRef] Shen, Dehua, Andrew Urquhart, and Pengfei Wang. 2019. Does twitter predict Bitcoin? Economics Letters 174: 118-22. [CrossRef]

Wei, Wang Chun. 2018. Liquidity and market efficiency in cryptocurrencies. Economics Letters 168: 21-24. [CrossRef]

(C) 2019 by the authors. Licensee MDPI, Basel, Switzerland. This article is an open access article distributed under the terms and conditions of the Creative Commons Attribution (CC BY) license (http://creativecommons.org/licenses/by/4.0/). 